\title{
Fermions on kinks revisited
}

\author{
Vladislav Klimashonok@ and Ilya Perapechka \\ Department of Theoretical Physics and Astrophysics, BSU, Minsk 220004, Belarus \\ Yakov Shnir@ \\ BLTP, JINR, Dubna 141980, Moscow Region, Russia
}

(Received 3 October 2019; published 6 November 2019)

\begin{abstract}
We study fermion modes localized on the kink in the $1+1$ dimensional $\phi^{4}$ model, coupled to the Dirac fermions with backreaction. Using numerical methods, we construct self-consistent solutions of the corresponding system of coupled integral-differential equations and study dependencies of the scalar field of the kink and the normalizable fermion bound states on the values of the parameters of the model. We show that the backreaction of the localized fermions significantly modifies the solutions, in particular it results in spatial oscillations of the profile of the kink and violations of the reflection symmetry of the configuration.
\end{abstract}

DOI: 10.1103/PhysRevD.100.105003

\section{INTRODUCTION}

Many nonlinear physical system support solitons, spatially localized field configurations with various ramifications for condensed matter physics, nonlinear optics, nuclear physics, cosmology, and quantum field theory [1-5]. Perhaps the simplest examples of solitons are the kinks which appear in models in one spatial dimension with a potential possessing two or more degenerated minima; see, e.g., $[6,7]$. Double well potential corresponds to the nonintegrable $\phi^{4}$ model; another interesting example are the kinks in the $\phi^{6}$ theory [8], kink-antikink collision in this model has attracted much attention recently $[9,10]$.

A peculiar feature of topological solitons is the relation between the topological charge of the configuration and the number of fermion zero modes localized on the soliton. The Atiyah-Patodi-Singer index theorem [11] yields a remarkable relation between these quantities.

Fermionic zero modes of the solitons were discussed first in the pioneering work [12], similar localized states exist on the vortices [13], domain walls [14], monopoles [15,16], sphalerons [17,18], and skyrmions [19-22]. The presence of localized fermion modes leads to some very interesting phenomena such as monopole catalysis of proton decay $[15,16]$, possible existence of superconducting cosmic strings [23], and appearance of fractional quantum numbers of solitons [13,24].

Published by the American Physical Society under the terms of the Creative Commons Attribution 4.0 International license. Further distribution of this work must maintain attribution to the author(s) and the published article's title, journal citation, and DOI. Funded by SCOAP ${ }^{3}$.
Fermions bounded by kinks were considered in many papers [24-28]. However, nearly all of the studies neglected the backreaction of the fermions on the soliton; moreover, only zero modes were considered in most cases. There has however been some attempt to take into account the backreaction of the fermion on the kink [29,30], although selfconsistent solution is still missing. One of the main reasons for that is the enormous computational complexity of the problem, there is no analytical solution of the corresponding system of coupled integral-differential equations.

A main objective of this paper is to reconsider this system consistently. Recently, we developed new numerical scheme which was successfully applied to examine the effects of backreaction of localized fermionic modes on planar skyrmions [31,32]. We have found that there is a tower of fermionic modes of two different types, localized by the soliton with one level crossing mode. Furthermore, in [32], we discussed a novel mechanism of exchange interaction between the skyrmions and constructed stable multisoliton configurations bounded by the attractive interaction mediated by the chargeless fermionic modes.

In the present paper, we revisit the fermion-kink bounded system with backreaction. Apart the well-known zero mode, which does not affect the kink for any values of the Yukawa coupling, we find various localized fermion modes with finite energy. The number of these bound modes increases as the Yukawa coupling becomes stronger, they are linked to the states of positive and negative continuum. We find that, as we increase the coupling, the effects of backreaction of the fermions on the kink becomes more and more significant. Furthermore, the localized fermions may give rise to additional exchange interaction between the solitons. 

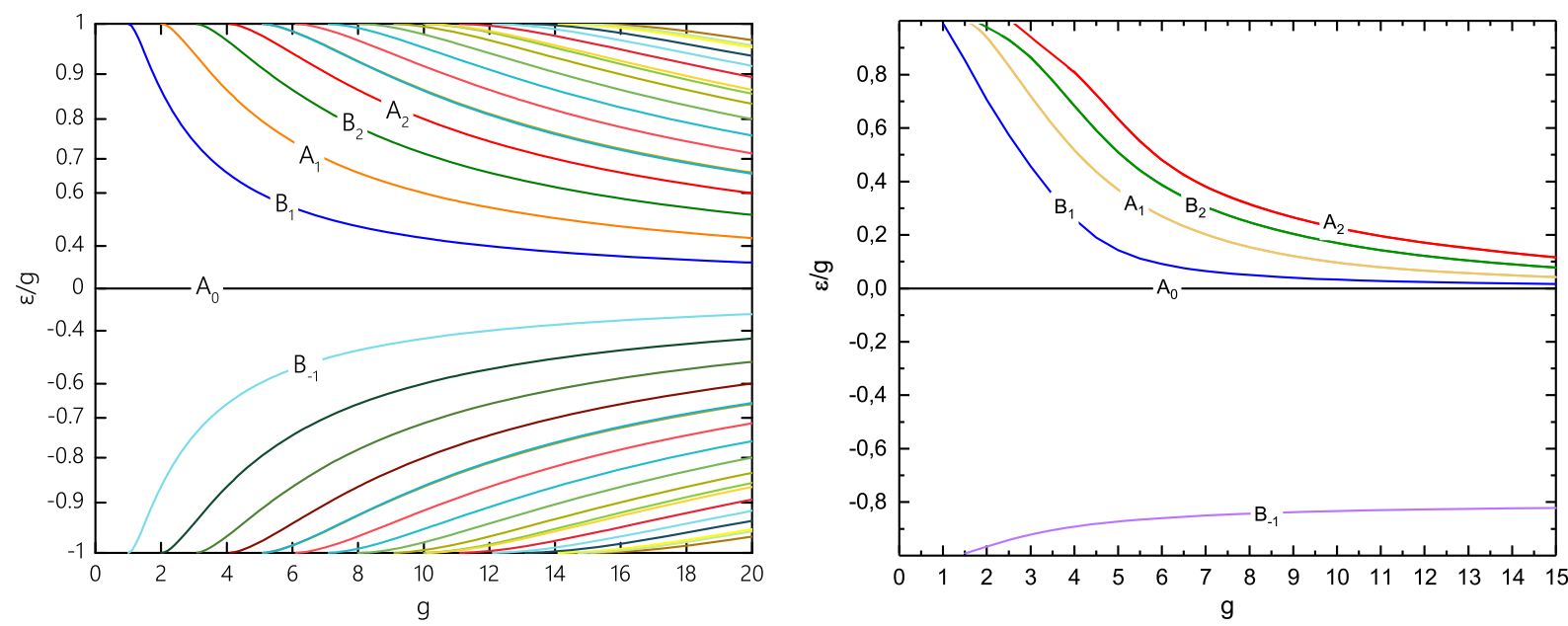

FIG. 1. Normalized energy $\frac{\epsilon}{g}$ of the localized fermionic states as a function of the Yukawa coupling $g$ for several fermion modes at $m=0$ without backreaction (left) and with backreaction of the fermions on the kink (right).

This paper is organized as follows. In Sec. II, we present the $\phi^{4}$ model coupled to Dirac fermions via the usual Yukawa coupling. Numerical results are presented in Sec. III, where we describe the solutions of the model and discuss the spectral flow of the localized fermionic states with backreaction on the kink. Conclusions and remarks are formulated in the last section.

\section{THE MODEL}

We consider a coupled fermion-scalar system in $1+1$ dimensions defined by the Lagrangian

$$
\mathcal{L}=\frac{1}{2} \partial_{\mu} \phi \partial^{\mu} \phi+\bar{\psi}\left[i \gamma^{\mu} \partial_{\mu}-m-g \phi\right] \psi-U(\phi),
$$

where $U(\phi)$ is a potential of the self-interacting scalar field, $\psi$ is a two-component spinor, and $m, g$ are the bare mass of the fermions and the dimensionful Yukawa coupling constant, respectively. The matrices $\gamma_{\mu}$ are $\gamma_{0}=\sigma_{1}, \gamma_{1}=$ $i \sigma_{3}$ where $\sigma_{i}$ are the Pauli matrices and $\bar{\psi}=\psi^{\dagger} \gamma^{0}$. The $\phi^{4}$ model corresponds to the quartic potential $U(\phi)=$ $\frac{1}{2}\left(1-\phi^{2}\right)^{2}$ with two vacua $\phi_{0} \in\{-1,1\}$.

The field equations of the system are given by

$$
\begin{aligned}
\partial_{\mu} \partial^{\mu} \phi+g \bar{\psi} \psi-2 \phi+2 \phi^{3} & =0, \\
i \gamma^{\mu} \partial_{\mu} \psi-m \psi-g \phi \psi & =0 .
\end{aligned}
$$

Using the usual parametrization of a two-component spinor

$$
\psi=e^{-i \epsilon t}\left(\begin{array}{l}
u(x) \\
v(x)
\end{array}\right),
$$

we obtain the following coupled system of static equations:

$$
\begin{aligned}
\phi_{x x}+2 g u v-2 \phi+2 \phi^{3} & =0, \\
u_{x}+(m+g \phi) u & =\epsilon v, \\
-v_{x}+(m+g \phi) v & =\epsilon u .
\end{aligned}
$$

This system is supplemented by the normalization condition $\int_{-\infty}^{\infty} d x\left(u^{2}+v^{2}\right)=1$, thus the configuration as a whole could can be characterized by two quantities, the fermionic density distribution $\rho_{f}=u^{2}+v^{2}$ and the topological density, i.e., the spatial derivative of the profile of the scalar field of the kink $\phi(x)$.

Note that the first equation in the system of dynamical equations (3) enjoys the reflection symmetries

$$
x \rightarrow-x, \quad \phi \rightarrow-\phi, \quad u v \rightarrow-u v,
$$

while the equations on the spinor components coupled to the scalar field are invariant with respect to the transformations

$$
x \rightarrow-x, \quad u \rightarrow v, \quad v \rightarrow u .
$$

Consideration of the fermionic modes is usually related with simplifying assumption that the scalar field background is fixed [24-28]. In the decoupled limit $g=0$, the $\phi^{4}$ model supports a spatially localized static topological soliton, the kink

$$
\phi_{K}(x)=\tanh \left(x-x_{0}\right)
$$

interpolating between the vacua $\phi_{0}=-1$ and $\phi_{0}=1$. Here $x_{0}$ is the position of the center of kink. The antikink solutions can be found by the inversion $x \rightarrow-x$. Clearly, the kink field is parity odd, it agrees with the symmetry condition (4). Then the reflection symmetry of the Dirac equation (5) means that the positive energy fermionic states localized on the kink are also the negative energy states localized on the antikink, and vice versa [26]. Further, due to this symmetry, there is only one zero mode of the Dirac 

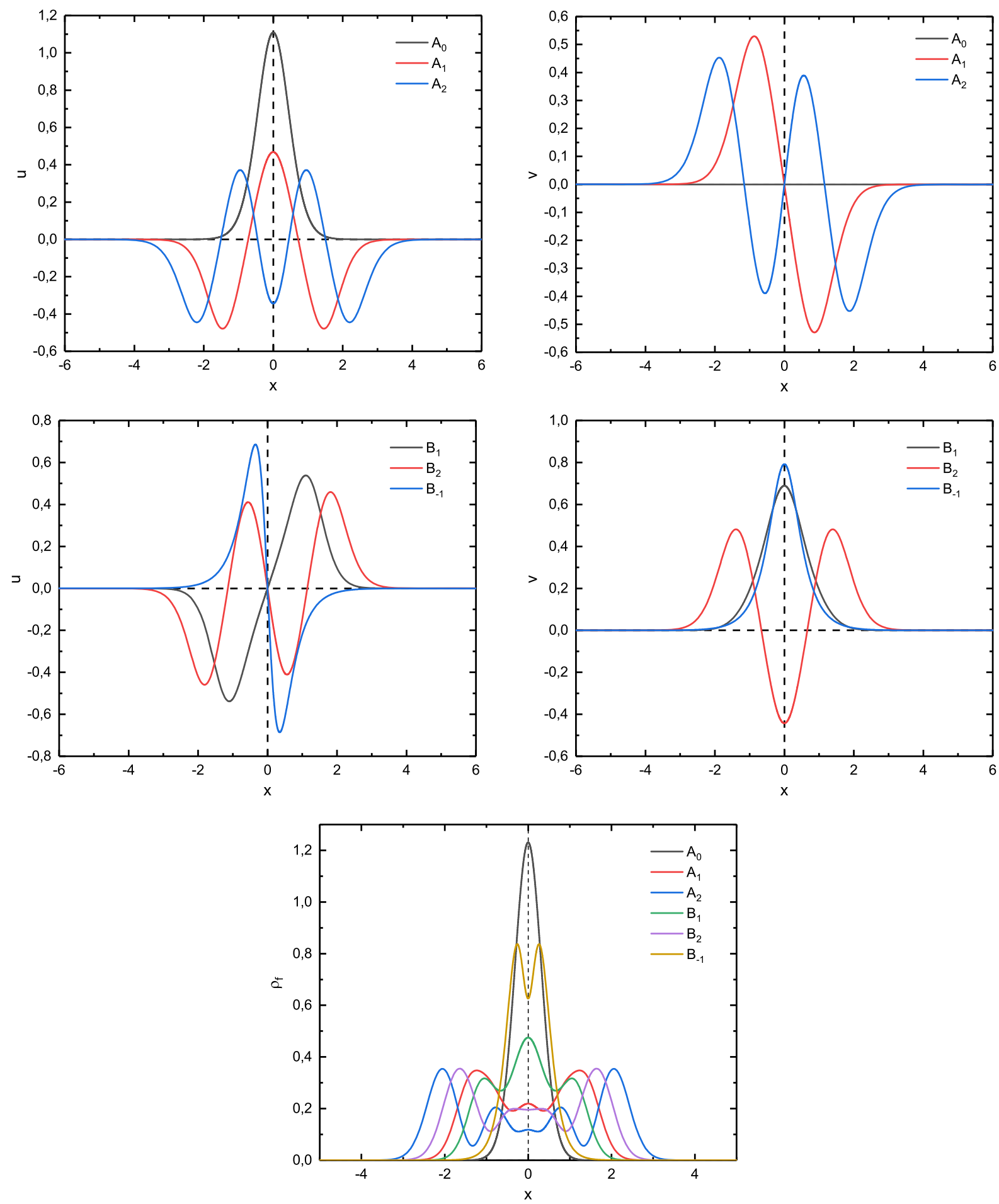

FIG. 2. Components of the localized fermionic modes of the types $A_{k}$ (upper row), $B_{k}$ (middle row), and the fermion density distribution of these modes (bottom plot) are plotted as functions of the coordinate $x$ for $m=0$ and $g=5$. Backreaction of the fermions on the kink is taken into account.

equation which is always a zero mode independently of the value of the Yukawa coupling $g$ [24-26],

$$
\psi_{0}=N_{0}\left(\begin{array}{c}
\frac{e^{-m x}}{\cosh ^{9} x} \\
0
\end{array}\right)
$$

where $N_{0}$ is a normalization factor. In the special case of the $N=1$ supersymmetric generalization of the model (1) [33], this mode is generated via the supersymmetry transformation of the boson field of the static kink. 

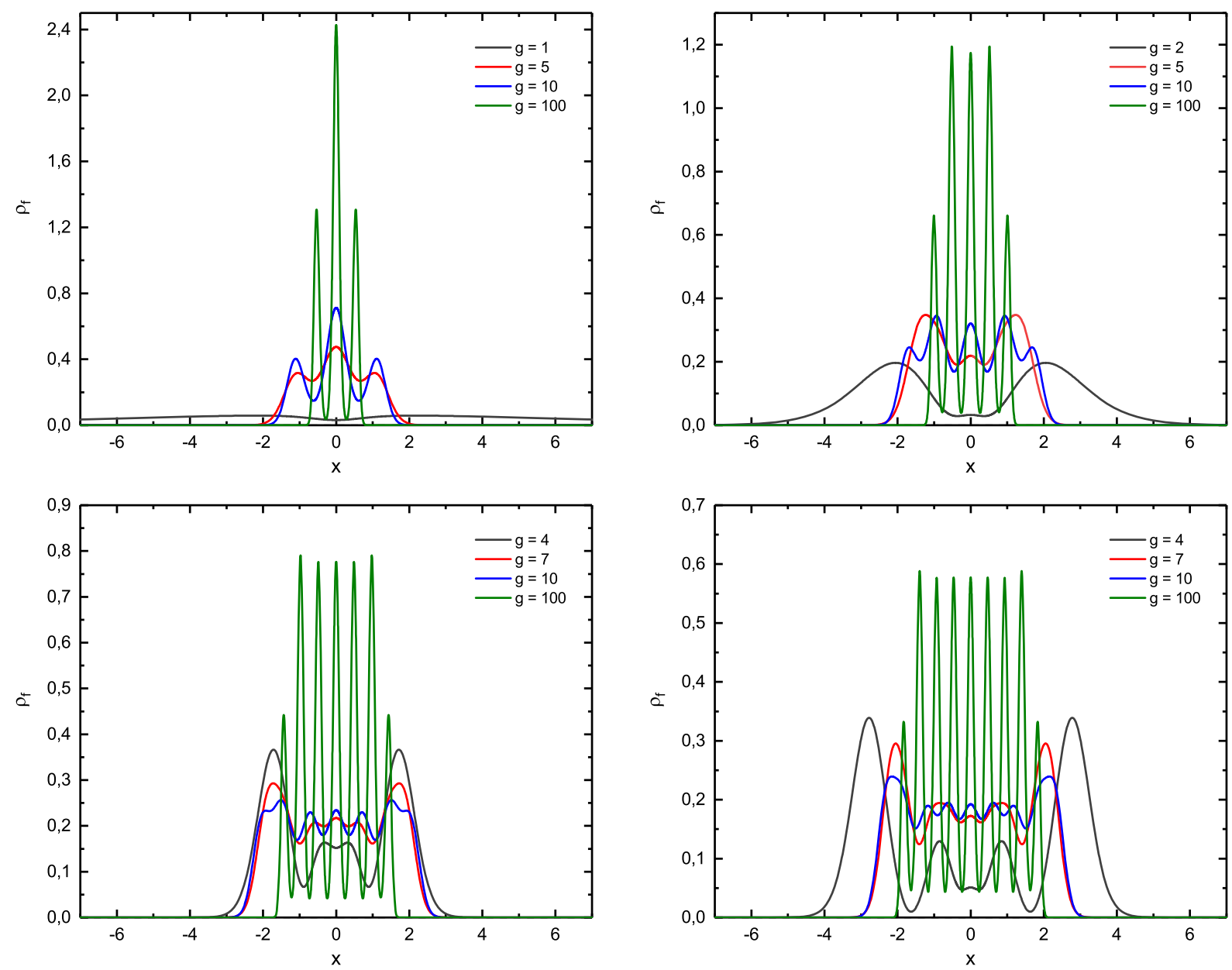

FIG. 3. The fermion density distributions of the localized modes $B_{1}$ (upper left plot), $A_{1}$ (upper right plot), $B_{2}$ (bottom left plot), and $A_{2}$ (bottom right plot) are plotted as functions of the coordinate $x$ for $m=0$ and several values of the Yukawa coupling $g$. Backreaction of the fermions on the kink is taken into account.

It was noticed that further increase of the Yukawa coupling $g$ gives rise to other fermion modes with nonzero energy, which are localized on the kink $[25,26]$. Indeed, the system of two first order differential equations in (3) can be transformed into two decoupled second order equations for the components $u$ and $v$ [25],

$$
\begin{aligned}
& -u_{x x}+\left((m+g \phi)^{2}-g \phi_{x}\right) u=\epsilon^{2} u, \\
& -v_{x x}+\left((m+g \phi)^{2}+g \phi_{x}\right) v=\epsilon^{2} v .
\end{aligned}
$$

They are Schrödinger-type equations, for the fermions in the external static background field of the kink (6), the corresponding potential is

$$
U_{f}=(m+g \tanh x)^{2} \pm \frac{g}{\cosh ^{2} x} .
$$

In the limit of zero bare mass of the fermions, $m=0$, the potential (9) becomes reduced to the usual Pöschl-Teller potential, so the equations (8) can be solved analytically $[25,26]$. Further, it was pointed out that as the Yukawa coupling $g$ increases, the potential well becomes deeper and new levels appear in the spectrum of the bound states. ${ }^{1}$ For example, there is a bound state solution for the massless fermions, which appears as the coupling increases above $g_{c r}=1$,

$$
\psi_{1}=N_{1}\left(\begin{array}{c}
\frac{ \pm \sqrt{2 g-1} \tanh x}{\cosh ^{g-1} x} \\
\frac{1}{\cosh ^{g-1} x}
\end{array}\right),
$$

with eigenvalues $\epsilon_{1}= \pm \sqrt{2 g-1}[26,29,34]$. Other solutions also can be written in a closed form; see [26]. The corresponding levels can be filled according to the

\footnotetext{
${ }^{1}$ Interestingly, for the given choice of the Yukawa coupling in (1), the corresponding equations for the fermions on the background of static kinks of the completely integrable sine-Gordon model, or for the fermions on the kinks of the $\phi^{6}$ model with triple vacuum $[8,9]$, do not support localized fermion states. However, the situation changes after an appropriate adjustment of the coupling [28], or modification of the scalar potential.
} 

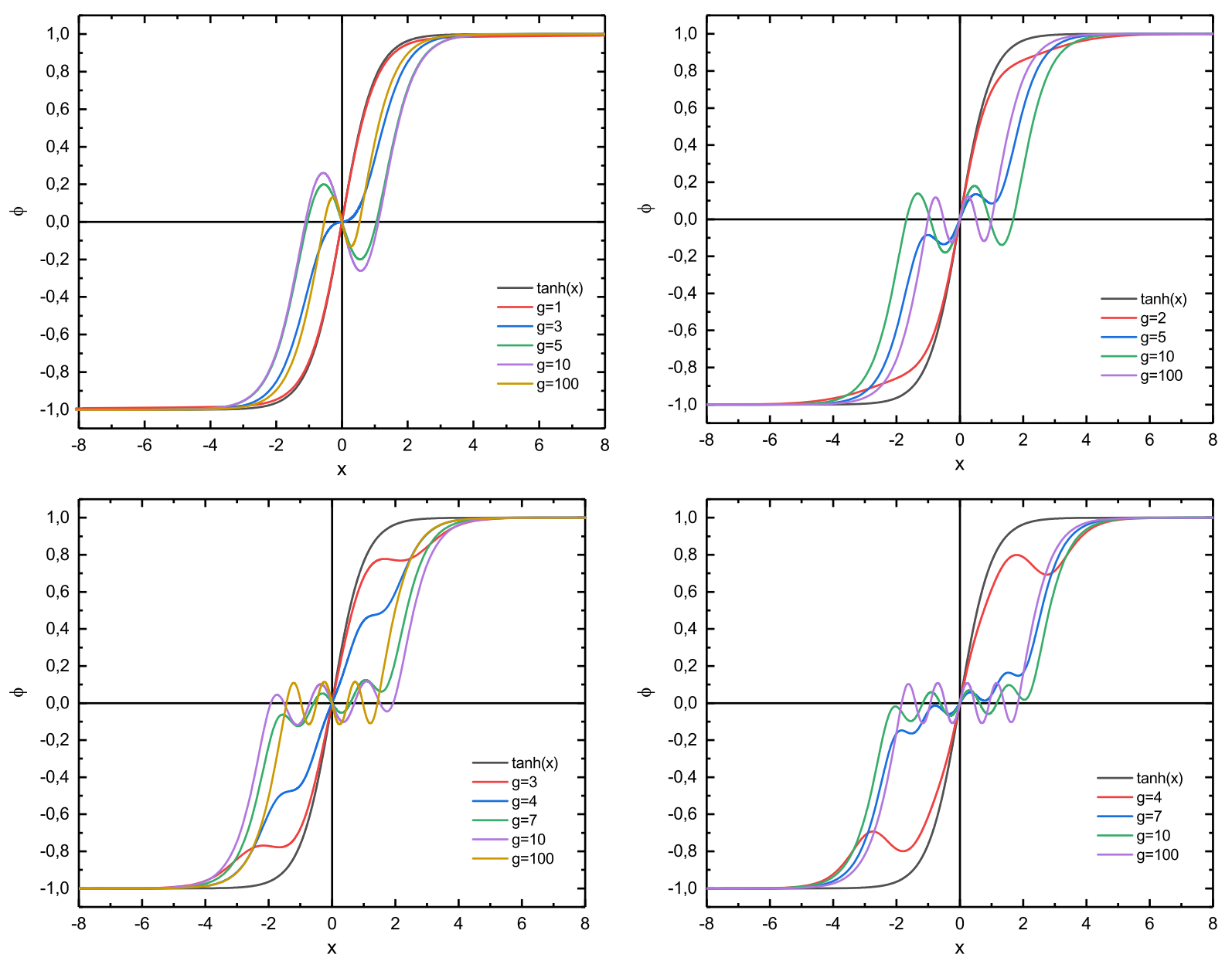

FIG. 4. The profiles of the scalar field of the kink, coupled to the localized fermionic modes $B_{1}$ (upper left plot), $A_{1}$ (upper right plot), $B_{2}$ (bottom left plot), and $A_{2}$ (bottom right plot) for $m=0$ and several values of the Yukawa coupling $g$.

Paulis exclusion principle, hereafter we restrict our consideration to the kink-fermion system with filling factor equal to 1 . We also made the usual assumption that the negative energy continuum (Dirac's sea) is filled.

Clearly, as the coupling becomes stronger, the backreaction of the bounded modes could significantly affect the scalar field, so the analytical solution for the fermion modes bounded by the kink is not quite correct for relatively large values of $g$. Indeed, as we will see below, in such a case the exact self-consistent numerical solutions of the coupled system of equations (2) become very different from the analytical results for the fermions in the external field of the static kink. Our goal here is to investigate this effect in a systematic way.

\section{NUMERICAL RESULTS}

We have solved numerically the full system of integraldifferential equation (2) with the normalization condition on the spinor field using eighth order finite-difference method. The system of equations is discretized on a uniform grid with usual size of 5000 points. To simplify our calculations, we consider only positive semi-infinite line taking into account the symmetry of the configuration (4), (5). Further, we map semi-infinite region onto the unit interval $[0,1]$ via the coordinate transformation $\tilde{x}=\frac{x}{c+x}$. Here $c$ is an arbitrary constant which is used to adjust the contraction of the grid. The emerging system of nonlinear algebraic equations is solved using a modified Newton method. The underlying linear system is solved with the Intel MKL PARDISO sparse direct solver. The errors are on the order of $10^{-9}$.

To obtain numerical solutions of the system (2), we have to impose appropriate boundary condition for the spinor field, both at the center of the kink and at the vacua. For fermions localized on the kink, we have to impose

$$
\begin{aligned}
& \left.\phi\right|_{-\infty}=-1,\left.\quad \phi\right|_{\infty}=1, \\
& \left.u\right|_{-\infty}=\left.u\right|_{\infty}=\left.v\right|_{-\infty}=\left.v\right|_{\infty}=0 .
\end{aligned}
$$

First, we consider the normalized fermions with zero bare mass $m=0$. Taking into account the symmetry 

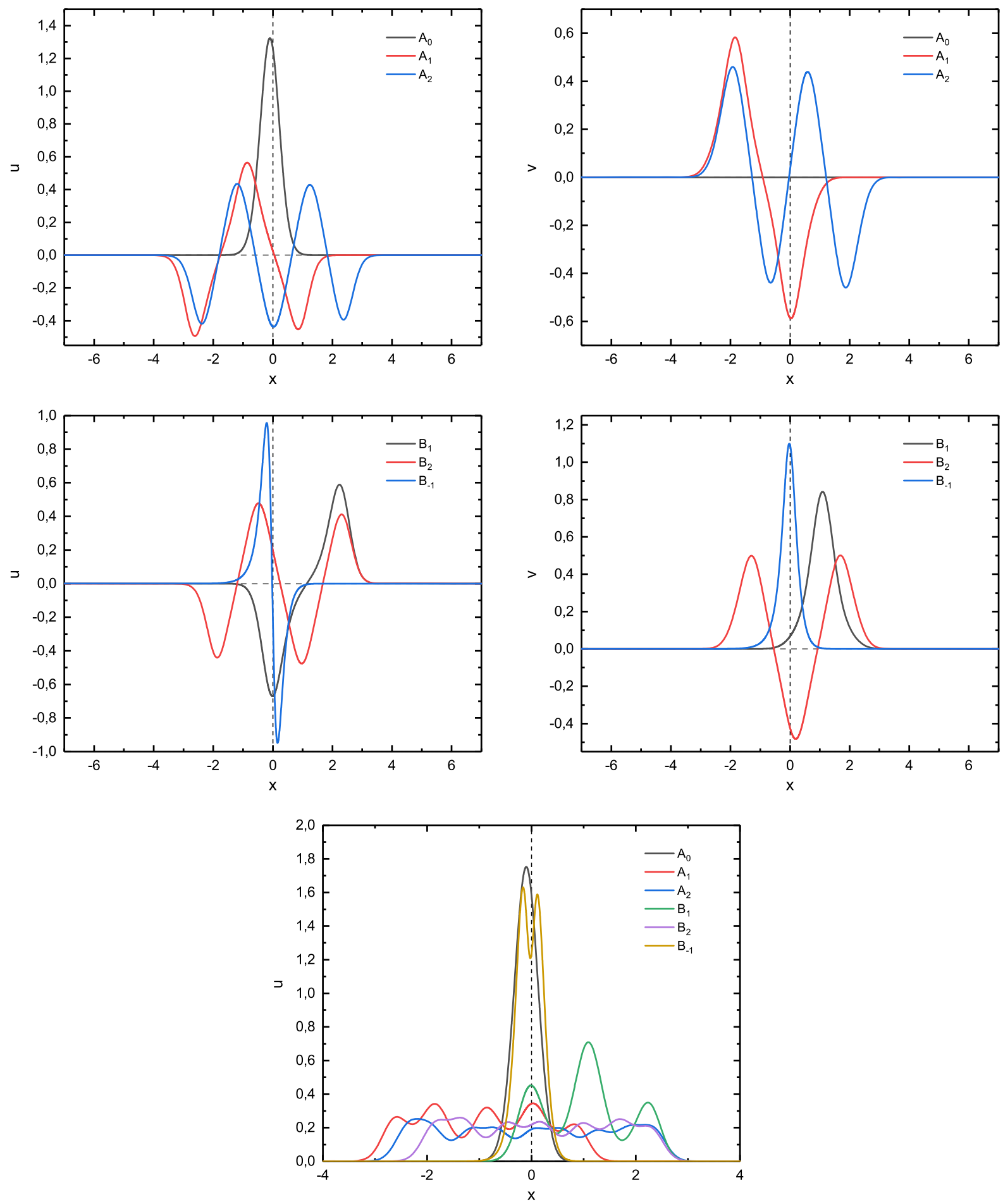

FIG. 5. Components of the localized fermionic modes of the types $A_{k}$ (upper row) and $B_{k}$ (middle row), and the fermion density distribution of these modes (bottom plot) are plotted as functions of the coordinate $x$ for $m=1$ and $g=10$. Backreaction of the fermions on the kink is taken into account.

properties (5) and the linearized equations (3) for the spinor field at $x=x_{0}=0$, we can classify the corresponding solutions according to their parity. Thus, we consider two types of the boundary conditions for the massless fermions at the center of the kink,

$$
\left.u_{x}\right|_{x_{0}}=\left.0 \quad v\right|_{x_{0}}=0 \quad \text { or }\left.\quad u\right|_{x_{0}}=\left.0 \quad v_{x}\right|_{x_{0}}=0 .
$$

We will refer to the modes of the first type to as $A_{k}$ modes and to the modes of the second tape to as $B_{k}$ modes, i.e., the modes of the type $A$ have symmetric $u$ component and 

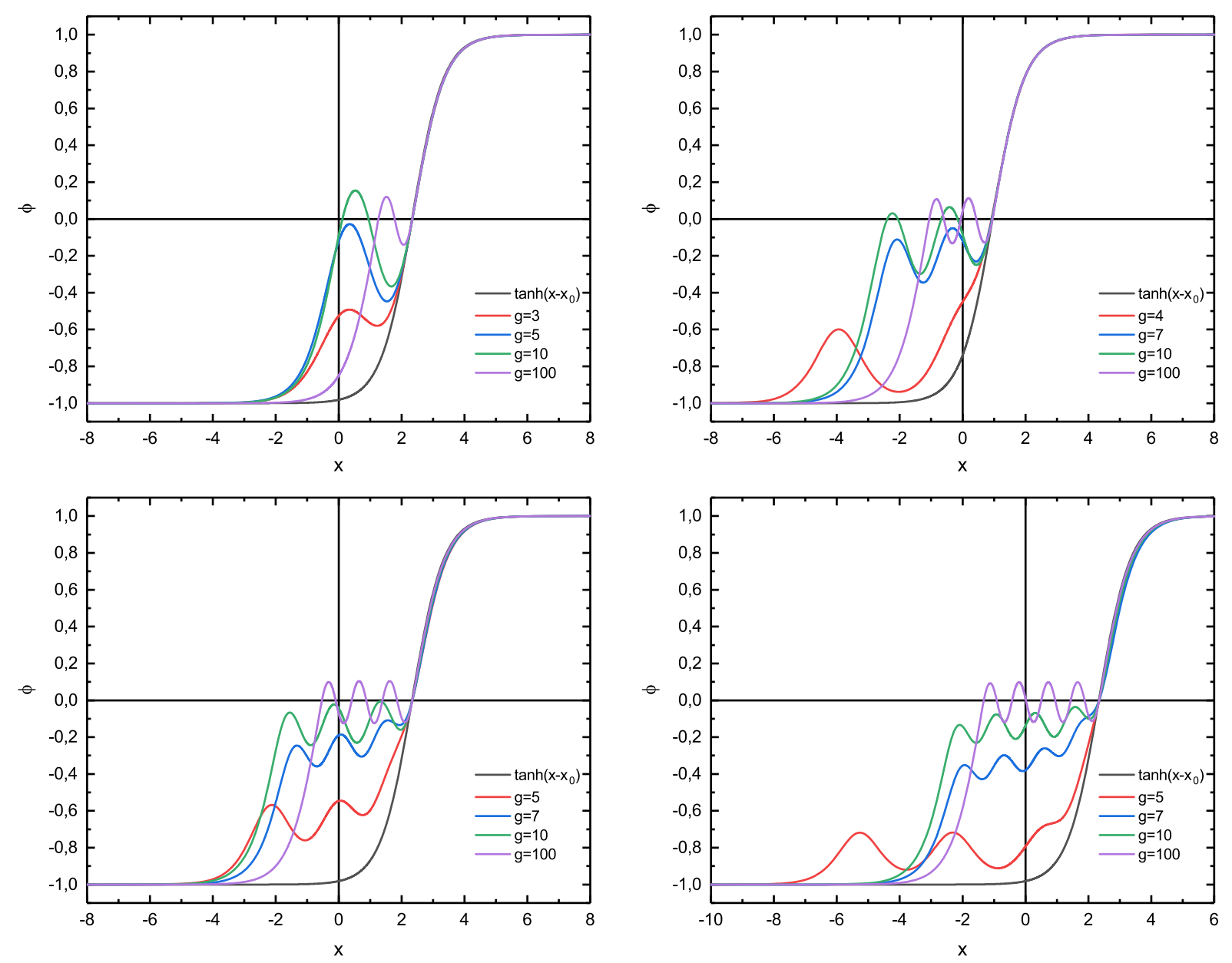

FIG. 6. The profiles of the scalar field of the kink, coupled to the localized fermionic modes $B_{1}$ (upper left plot), $A_{1}$ (upper right plot), $B_{2}$ (bottom left plot), and $A_{2}$ (bottom right plot) for $m=1$ and several values of the Yukawa coupling $g$.

antisymmetric $v$ component, while the modes of the type $B$ have antisymmetric $u$ component and symmetric $v$ component. Here, the index $k$ corresponds to the minimal number of nodes of the components; for example, the zero mode (7) is denoted as $A_{0}$. Note that for all modes, the number of nodes of the component $u$ is one node more than the number of nodes of the component $v$.

In the decoupling limit, the backreaction of the fermions on the kink is neglected, the pair of the first order equations on the spinor components in the system (2) describes the fermion states in the external scalar field of kink $\phi_{K}(x)$ (6). In such a case, the energy spectrum of the localized fermions is symmetric with respect to inversion $\epsilon \rightarrow-\epsilon$, apart zero mode $A_{0}$, each state with a positive eigenvalue $\epsilon$ has a counterpart with reflected antisymmetric $u(v)$ component and a negative eigenvalue $-\epsilon$; see Fig. 1, left plot.

The situation is very different in the full coupled system (2) with backreaction, the profile of the kink deforms as a fermion occupies an energy level. Further, the energy levels of the bounded fermions move accordingly and the symmetry between the localized fermion states with positive and negative eigenvalues $\epsilon$ is violated; see Fig. 1, right plot.

Considering the spectral flow of the localized fermions, we observe that in the range of values of the coupling $0<g<1$ there is only one localized zero mode, as seen in Fig. 1. As the coupling $g$ grows, we obtain an infinite tower of new solutions of the model (1) which correspond to the states of deformed $\phi^{4}$ kink with different types and filling factors of localized fermions with nonzero eigenvalues $\epsilon$. The deformation of the coupled configuration drives the nonzero eigenvalues $\epsilon$ of all modes toward negative energy continuum; see Fig. 1, right plot.

In Fig. 2, we display the components of a few modes of both types, localized on the kink with backreaction, and the corresponding distributions of the fermionic density $\rho_{f}(x)$.

In Fig. 3, we plot the fermionic density distributions $\rho_{f}$ of the first four localizing modes $A_{1}, A_{2}, B_{1}, B_{2}$ with nonzero energy for several values of coupling constant $g$. At small values of the coupling constant $g$, there is only one localizing mode $A_{0}$ which should exists according to the index theorem. As the coupling $g$ increases, more modes of both types become localized by the kink. 

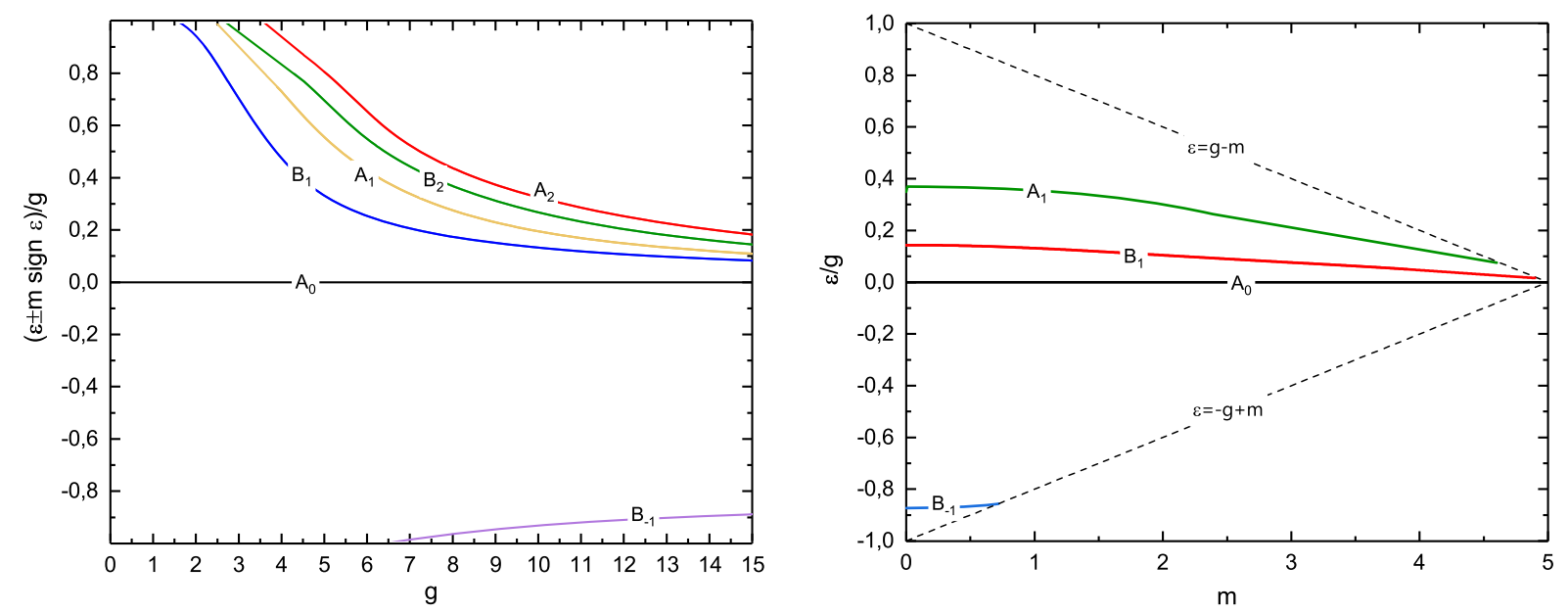

FIG. 7. Normalized energy $\frac{\epsilon+m \operatorname{sign} \epsilon}{g}$ of the localized fermionic states as a function of the Yukawa coupling $g$ for several fermion modes at $m=1$ (left plot) and the normalized spectral flow $\epsilon / g$ of the massive localized fermions as a function of the bare mass $m$ (right plot).

Further increase of the coupling $g$ yields stronger bounding of the modes, also larger number of localized modes are extracted from the positive and negative continuum.

The effect of backreaction of the fermions coupled to the kink is illustrated in Fig. 4. As expected, the massless zero mode does not distort the kink for any values of the Yukawa coupling. However, the scalar field is strongly affected by other bounded modes with nonzero energy. For example, it is seen in Fig. 4, left upper plot, that the coupling of the kink to the mode $B_{1}$ leads to distortion of the profile of the soliton, which closely resembles the deformation of the kink due to excitation of its normalizable discrete vibrational mode; see, e.g., $[4,7,35]$. Clearly, by analogy with excitations of this internal mode of the kink [36], dynamical coupling to the fermions may lead to production of the kink-antikink pairs.

Coupling of the kink to the modes with some number of nodes is reflected in visible spatial oscillations of the static scalar field at the center of the kink, where fermion modes are located; see Fig. 4. In some sense, this configuration can be thought of as a chain of kink-antikink pairs tightly bounded by the localized fermions. Clearly, the coupling to higher fermionic modes yields much stronger deformations of the kink.

Note that the deformations of the kink caused by its coupling with higher fermion modes at strong coupling look similar to the system of iterated kinks in a model with impurities, considered in [37]. Indeed, in a certain sense, the Yukawa coupling to the fermions acts like an impurity in the $\phi^{4}$ system.

Next, we consider dependence of solutions on the value of the fermion bare mass $m$. In that case, the energy of the localized fermionic states is restricted as $|\varepsilon|<|g-m|$. Note that, even for the fermions localized on the kink without the backreaction, the bare mass term violates the reflection symmetry of the equations (8), the term $m+g \tanh x$ does not have a definite parity. Indeed, our numerical simulations confirm that for nonzero values of the bare mass $m$, both the scalar field of the kink and fermionic densities of localized modes are asymmetric; see Figs. 5 and 6 where we display numerical solution of the system (3) at $m=1$ for a fixed value of the coupling constant $g$. Further increase of the fermion mass stronger deforms the configuration, the size of the deformed area increases.

The massive mode $A_{0}$ also becomes more localized; however, corresponding eigenvalue remains zero and there is no zero-crossing mode in the spectral flow of the massive fermions coupled to the kink in $1+1$ dimensional system. The spectrum of the massive fermions is not very different from the massless case above; we can see it comparing Figs. 1 and 7, left plot. For a fixed value of the Yukawa coupling, the massive modes delocalize at some critical values of the fermion mass $m$; see Fig. 7, right plot.

\section{SUMMARY AND CONCLUSIONS}

In this paper, we present a novel self-consistent approach of analyzing fermionic states localized on the kink in the $\phi^{4}$ theory taking into account the deformations of the soliton due to the presence of bounded fermions. The full system of coupled field equations for the real scalar field and the Dirac fermions coupled to the kink via the usual Yukawa coupling is supplemented by the normalization condition for the localized fermions. For imposing appropriate boundary conditions both for scalar and spinor fields we constructed numerical solutions of the resulting system of integral-differential equations and found the corresponding energy eigenvalues. Apart the usual zero mode, which does not affect the kink for any values of the coupling and the bare mass $m$, there is a tower of localized states with nonzero eigenvalues, they are linked to the positive and negative continuum. 
We have shown that the backreaction of the fermions strongly affects the spectrum; it breaks the symmetry between the localized modes with positive and negative eigenvalues. Further, the refection symmetry of the usual Dirac equation on static kink background is violated for the fermions with nonzero bare mass; these spinors do not possess definite parity.

Localization of the fermions on the kink yields spatial oscillations of the static scalar field at the center of the kink, where fermion modes are located. This configuration can be thought of as a chain of kink-antikink pairs tightly bounded by the localized fermions; the number of the constituents on the chain increases for higher fermionic modes.

The work here should be taken further by considering fermionic states localized on various topological solitons with backreaction. In particular, it would be interesting to study the effects of the fermionic modes localized on nonBPS monopoles and on the Abelian vortices and the corresponding superconducting strings. Another interesting question, which we hope to be addressing in the near future, is to investigate the exchange interaction between the solitons mediated by the fermions, a first step in this direction has been made in [32].

\section{ACKNOWLEDGMENTS}

Y.S. would like to thank Steffen Krusch, Tomasz Romańczukiewicz, Tanmay Vachaspati, and Andrzej Wereszczyński for enlightening discussions. He gratefully acknowledges partial support of the Ministry of Science and Higher Education of Russian Federation, Project No. 3.1386.2017.
[1] Solitons and Condensed Matter Physics, edited by A. R. Bishop and T. Schneider (Springer-Verlag, Berlin, 1978).

[2] T. Dauxois and M. Peyrard, Physics of Solitons, (Cambridge University Press, Cambridge, United Kingdom, 2006).

[3] N.S. Manton and P. Sutcliffe, Topological Solitons (Cambridge University Press, Cambridge, United Kingdom, 2004).

[4] Y. M. Shnir, Topological and Non-Topological Solitons in Scalar Field Theories (Cambridge University Press, Cambridge, United Kingdom, 2018).

[5] A. Vilenkin and E. P. S. Shellard, Cosmic Strings and Other Topological Defects (Cambridge University Press, Cambridge, United Kingdom, 1994).

[6] T. Vachaspati, Kinks and Domain Walls (Cambridge University Press, Cambridge, United Kingdom, 2006).

[7] A Dynamical Perspective on the $\phi^{4}$ Model: Past, Present and Future, edited by P. G. Kevrekidis and J. CuevasMaraver (Springer, New York, 2019).

[8] M. A. Lohe, Phys. Rev. D 20, 3120 (1979).

[9] P. Dorey, K. Mersh, T. Romanczukiewicz, and Y. Shnir, Phys. Rev. Lett. 107, 091602 (2011).

[10] V. A. Gani, A. E. Kudryavtsev, and M. A. Lizunova, Phys. Rev. D 89, 125009 (2014).

[11] M. F. Atiyah, V. K. Patodi, and I. M. Singer, Math. Proc. Cambridge Philos. Soc. 77, 43 (1975).

[12] C. Caroli, P. G. De Gennes, and J. Matricon, Phys. Lett. 9, 307 (1964).

[13] R. Jackiw and P. Rossi, Nucl. Phys. B190, 681 (1981).

[14] D. Stojkovic, Phys. Rev. D 63, 025010 (2000).

[15] V. A. Rubakov, Nucl. Phys. B203, 311 (1982).
[16] C. G. Callan, Jr., Phys. Rev. D 26, 2058 (1982).

[17] C. R. Nohl, Phys. Rev. D 12, 1840 (1975).

[18] J. Boguta and J. Kunz, Phys. Lett. 154B, 407 (1985).

[19] J. R. Hiller and T. F. Jordan, Phys. Rev. D 34, 1176 (1986).

[20] S. Kahana, G. Ripka, and V. Soni, Nucl. Phys. A415, 351 (1984).

[21] S. Kahana and G. Ripka, Nucl. Phys. A429, 462 (1984).

[22] G. Ripka and S. Kahana, Phys. Lett. 155B, 327 (1985).

[23] E. Witten, Nucl. Phys. B249, 557 (1985).

[24] R. Jackiw and C. Rebbi, Phys. Rev. D 13, 3398 (1976).

[25] R. F. Dashen, B. Hasslacher, and A. Neveu, Phys. Rev. D 10, 4130 (1974).

[26] Y.Z. Chu and T. Vachaspati, Phys. Rev. D 77, 025006 (2008).

[27] Y. X. Liu, L. D. Zhang, L. J. Zhang, and Y. S. Duan, Phys. Rev. D 78, 065025 (2008).

[28] Y. Brihaye and T. Delsate, Phys. Rev. D 78, 025014 (2008).

[29] V. A. Gani, V. G. Ksenzov, and A. E. Kudryavtsev, Phys. At. Nucl. 73, 1889 (2010).

[30] A. Amado and A. Mohammadi, Eur. Phys. J. C 77, 465 (2017).

[31] I. Perapechka, N. Sawado, and Y. Shnir, J. High Energy Phys. 10 (2018) 081.

[32] I. Perapechka and Y. Shnir, Phys. Rev. D 99, 125001 (2019).

[33] P. Di Vecchia and S. Ferrara, Nucl. Phys. B130, 93 (1977).

[34] M. Postma and B. Hartmann, arXiv:0706.0416.

[35] R. Rajaraman, Phys. Rep. 21, 227 (1975).

[36] N. S. Manton and H. Merabet, Nonlinearity 10, 3 (1997).

[37] N. S. Manton, K. Oles, and A. Wereszczynski, J. High Energy Phys. 10 (2019) 086. 